ARTICLE

DOI: $10.1038 / \mathrm{s} 41467-018-03536-\mathrm{x}$

\title{
Structure of the activated Edc1-Dcp1-Dcp2-Edc3 mRNA decapping complex with substrate analog poised for catalysis
}

Jeffrey S. Mugridge ${ }^{1}$, Ryan W. Tibble ${ }^{1,2}$, Marcin Ziemniak ${ }^{3,4,5}$, Jacek Jemielity ${ }^{4}$ \& John D. Gross (1) ${ }^{1,2}$

The conserved decapping enzyme Dcp2 recognizes and removes the $5^{\prime}$ eukaryotic cap from mRNA transcripts in a critical step of many cellular RNA decay pathways. Dcp2 is a dynamic enzyme that functions in concert with the essential activator Dcp1 and a diverse set of coactivators to selectively and efficiently decap target mRNAs in the cell. Here we present a $2.84 \AA$ crystal structure of $K$. lactis Dcp1-Dcp2 in complex with coactivators Edc1 and Edc3, and with substrate analog bound to the Dcp2 active site. Our structure shows how Dcp2 recognizes cap substrate in the catalytically active conformation of the enzyme, and how coactivator Edc1 forms a three-way interface that bridges the domains of Dcp2 to consolidate the active conformation. Kinetic data reveal Dcp2 has selectivity for the first transcribed nucleotide during the catalytic step. The heterotetrameric Edc1-Dcp1-Dcp2-Edc3 structure shows how coactivators Edc1 and Edc3 can act simultaneously to activate decapping catalysis.

\footnotetext{
${ }^{1}$ Department of Pharmaceutical Chemistry, University of California, San Francisco, San Francisco, CA 94158, USA. ${ }^{2}$ Program in Chemistry and Chemical Biology, University of California, San Francisco, San Francisco, CA 94158, USA. ${ }^{3}$ Division of Biophysics, Institute of Experimental Physics, Faculty of Physics, University of Warsaw, 02-089 Warsaw, Poland. ${ }^{4}$ Centre of New Technologies, University of Warsaw, 02-097 Warsaw, Poland. ${ }^{5}$ Present address: Biological and Chemical Research Centre, Department of Chemistry, University of Warsaw, 02-089 Warsaw, Poland. Correspondence and requests for materials should be addressed to J.D.G. (email: jdgross@cgl.ucsf.edu)
} 
D egradation of mRNA transcripts plays a critical role in maintenance of the cellular transcriptome and in control of eukaryotic gene expression ${ }^{1}$. Removal of the $\mathrm{m}^{7} \mathrm{GpppN}$ cap structure found at the $5^{\prime}$ end of all eukaryotic mRNA marks these transcripts for rapid clearance via the $5^{\prime}$-to- $3^{\prime}$ mRNA decay pathway ${ }^{2}$. The conserved mRNA-decapping enzyme Dcp2 catalyzes hydrolysis of the $5^{\prime}$ cap to liberate $\mathrm{m}^{7} \mathrm{GDP}$ and $5^{\prime}$ monophosphate RNA, which can then be degraded by conserved $5^{\prime}-3^{\prime}$ exonucleases $^{3-6}$. Dcp2-mediated decapping is a critical step in many cellular RNA decay pathways including bulk $5^{\prime}-3^{\prime}$ decay 4,5 , nonsense-mediated decay $^{7-10}$, microRNA-mediated decay ${ }^{11,12}$, decay of long-noncoding RNA ${ }^{13}$, and decay of transcripts containing non-optimal codons ${ }^{14}$. Recently, Dcp2 was also shown to be a reader of reversible $\mathrm{m}^{6} \mathrm{~A}$ modifications in the $5^{\prime}$ cap, whereby $\mathrm{m}^{6} \mathrm{~A}$ methylation inhibits decapping and stabilizes cellular mRNA transcripts ${ }^{15}$.

Dcp 2 consists of an N-terminal regulatory domain (NRD) and a catalytic Nudix domain $(\mathrm{CD})^{16,17}$ linked by a short, flexible hinge that allows the enzyme to dynamically adopt different conformations in solution ${ }^{18,19}$ and in crystal structures ${ }^{20-24}$. The CD harbors a positively charged patch for RNA binding (box B motif) ${ }^{25,26}$ and a Nudix motif with conserved Glu residues that bind magnesium and carry out cap hydrolysis chemistry ${ }^{27,28}$. Fungal Dcp2 also contains an unstructured C-terminal extension that harbors protein-protein interaction motifs and elements that inhibit Dcp2 catalysis ${ }^{29}$. At least some of these motifs are transferred to the C-terminus of the essential activator Dcpl in metazoans, suggesting a conserved biological function ${ }^{30}$. The $\mathrm{NRD}$ enhances decapping activity by specifically recognizing the $\mathrm{m}^{7} \mathrm{G}$ nucleotide of cap and binding Dcp1, which further accelerates decapping $17,19,21,22,31,32$.

The activity of the core Dcp1-Dcp2 complex can be further modulated by the enhancer of decapping proteins (Edcs) ${ }^{33}$. For example, the Edcl-type activators, which include the yeast paralog Edc2 and PNRC2 in metazoans, contain conserved tandem short linear motifs to bind Dcp1 and activate Dcp2 catalysis ${ }^{19-21,34-37}$. Edc3 activates decapping by promoting RNA binding and alleviating autoinhibition of Dcp2 in yeast ${ }^{22,29,38-42}$. Edc3 is recruited to the decapping complex via short linear motifs found in the fungal or metazoan Dcp2 or Dcp1 C-terminal extension, respectively ${ }^{29,33}$. Edc4 is a metazoan-specific scaffolding protein that strengthens Dcp1-Dcp2 interaction and coordinates decapping with exonucleolytic degradation by Xrn $1^{32,43-45}$. Genetic and physical interactions between activator proteins suggest these cofactors work together to promote decapping, but how this is achieved at the structural level is unknown $^{45-49}$

Several recent structural studies have provided new insights into how Dcp2 recognizes the $\mathrm{m} 7 \mathrm{G}$ cap, how Dcp2 activity is enhanced by coactivators, and how Dcp2 solution conformations are tied to catalysis. First, a study by Valkov et al. presented a structure of $S$. pombe Edc1-Dcp1-Dcp2 in which the activation motif in Edc1 bridges the Dcp2 NRD and CD to apparently promote a conformation of Dcp 2 that enhances RNA binding ${ }^{20}$. Soon after, our lab published the first substrate analog-bound structure of $S$. pombe Dcp1-Dcp2 in complex with human $\mathrm{PNRC}^{21}$. Our structure showed a very different conformation than the Valkov structure, in which the substrate analog was bound by a composite binding site using conserved residues on the NRD and CD. However, in this pre-catalytic conformation of Dcp2 the catalytic Glu on the Nudix helix was positioned too far from substrate for effective catalysis, and the activation motif of PNRC2 was disordered, suggesting an additional conformational change in Dcp2 was needed to achieve catalysis. Simultaneously, Charenton et al. published a K. lactis Dcp1-Dcp2-Edc3 structure with bound $\mathrm{m}^{7} \mathrm{GDP}$ product in which $\mathrm{m}^{7} \mathrm{G}$ was recognized by the
Dcp2 NRD as in our Mugridge et al. substrate-bound structure, but the $C D$ was rotated by $\sim 90^{\circ}$ to bring the Nudix helix much closer to the cleaved $\mathrm{m}^{7} \mathrm{GDP}$ product, consistent with an active conformation of Dcp $2^{22}$. Finally, most recently Wurm et al. published a study that presented an S. pombe Edc1-Dcp1-Dcp2 product-bound structure in the same conformation as observed by Charenton et al., a conformation that is significantly populated in solution when Edcl and $\mathrm{m}^{7} \mathrm{GDP}$ or capped RNA are bound ${ }^{19}$.

In this study, we present a new, four-protein structure of $K$. lactis Edc1-Dcp1-Dcp2-Edc3 at $2.84 \AA$ resolution in the catalytically active conformation with bound substrate analog poised for catalysis. We show that the conserved YAGxxF activation motif of Edc1 mediates a three-way interface between Dcp1 and the Dcp2 NRD and CD, and that lesions on the NRD and in the flexible hinge linking the $\mathrm{CD}$ and NRD abolish activation by Edc1. Substrate analog binding to the active conformation of Dcp2 suggests that the first transcribed nucleotide of RNA is recognized by base stacking to a conserved aromatic residue at the top of the RNA-binding channel, and that the RNA body follows this positively charged path to bound Edc3 coactivator. We show that Dcp2 has selectivity for the first transcribed nucleotide of RNA substrate and that this selectivity depends on the conserved aromatic residue that binds this nucleotide of our substrate analog. Overall, these data show how substrate binds and is recognized by the catalytically active conformation of Dcp2, suggest a path for RNA binding consistent with prior in vitro mutagenesis and binding data, and show how coactivators Edc1 and Edc3 can simultaneously bind Dcp2 to enhance mRNA decapping by the Dcp1-Dcp2 complex. Our structure establishes that the active conformation of Dcp2 is the same for both substrate and product-bound complexes, and clarifies the mechanisms of decapping catalysis and control of decapping activation by multiple protein interactions.

\section{Results}

Structure of substrate analog-bound Edc1-Dcp1-Dcp2-Edc3. To obtain our recently published substrate analog-bound PNRC2-Dcp1-Dcp2 structure ${ }^{21}$, we crystallized an apo PNRC2-Dcp1-Dcp2 complex and soaked in cap analog to induce a conformational change and substrate binding within the crystal. While this substrate-bound conformation gave new insight into cap recognition by Dcp2, it was clear that this did not represent the catalytically active conformation because the catalytic Glu residue on the Nudix helix was positioned to too far from bound substrate to carry out hydrolysis chemistry. When we attempted to push Dcp2 into the active conformation by soaking in catalytically required magnesium along with substrate analog, the crystals were physically damaged and diffraction was completely destroyed, suggesting this induced an additional conformational change in Dcp2 that could not be accommodated by the crystal lattice.

To trap the substrate-bound active conformation, we screened different Dcp1-Dcp2 constructs (S. pombe, K. lactis, H. sapiens) in which the catalytic Glu was mutated to Gln to prevent cap hydrolysis for co-crystallization with magnesium, a tight-binding two-headed cap analog 50 , and coactivators Edc1 and Edc3 (Fig. 1a,b). The K. lactis constructs crystallized most readily and we obtained a structure of $K l$ Edc1-Dcp1-Dcp2-Edc3 with bound magnesium and two-headed cap analog at $2.84 \AA$ resolution (Fig. 1c, Table 1, solved by molecular replacement with PDB 5LOP 22 Dcp1-Dcp2). Dcp2 is in the catalytically active conformation, very similar to that found in recently reported $\mathrm{m}^{7}$ GDP product-bound structures (Supplementary Fig. 1; Dcp2 core backbone RMSD is $1.2 \AA$ with PDB 5LOP and $1.9 \AA$ with PDB 5N2V) ${ }^{19,22}$, with the Nudix helix positioned close to bound 
a<smiles>C[n+]1cn(C2OC(COP(=O)([O-])OP(=O)([O-])OP(=O)([O-])OP(=O)([O-])OP(=O)([O-])O)C(O)C2O)c2nc(N)[nH]c(=O)c21</smiles>

b

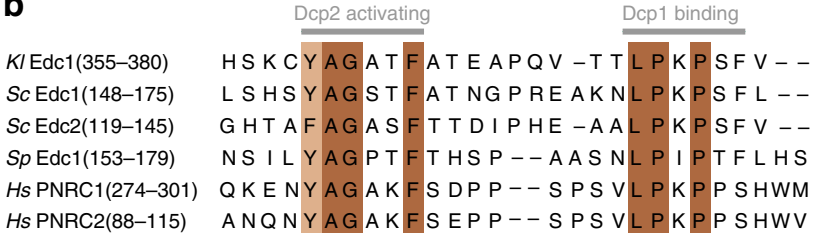

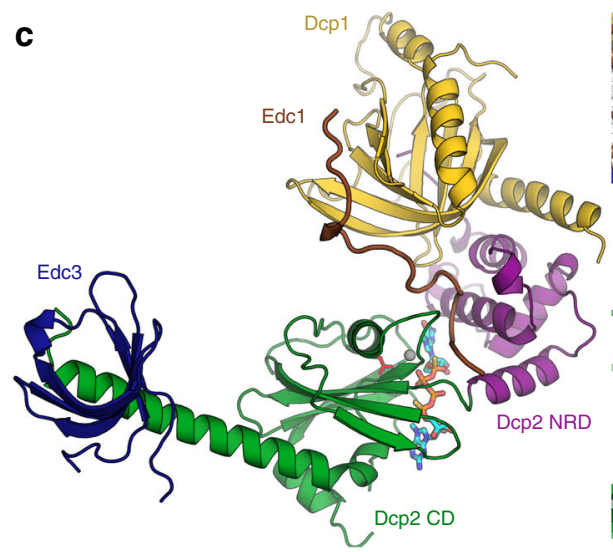
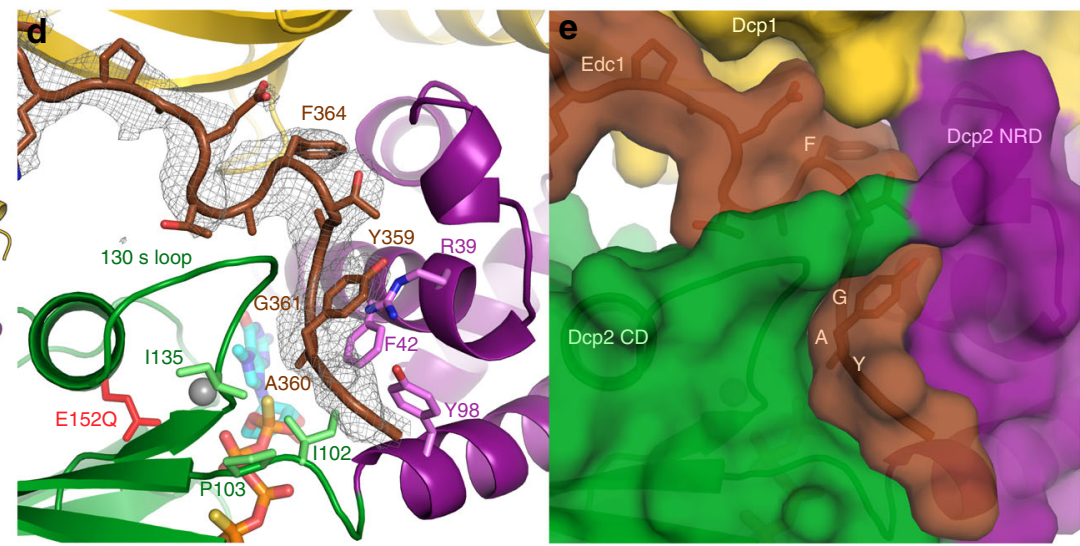

Fig. 1 Structure of the activated decapping complex poised for catalysis. a Structure of the high-affinity, two-headed cap substrate analog. $\mathbf{b}$ Sequence alignment of Edc1-like coactivator peptides showing the conserved Dcp2-activating and Dcp1-binding motifs; background color intensity is scaled to

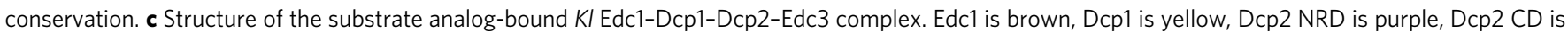
green, Edc3 Lsm domain is dark blue, substrate analog is cyan. d Close-up view of the three-way interface between Dcp1, Dcp2 NRD, and Dcp2 CD mediated by the Edc1 YAGxxF Dcp2-activating motif. $F_{\mathrm{o}}-F_{\mathrm{c}}$ omit map for the Edc1 peptide is shown at $2.5 \sigma$. Sidechains for the Edc1 peptide and nearby conserved residues on Dcp2 are shown as sticks. The catalytic Glu on the Nudix helix, mutated in our structure to GIn, is labeled as E152Q in red. e Surface view of the Edc1 activation motif-Dcp1-Dcp2 interface, with conserved residues in the activation motif labeled

cap. Edc3 Lsm domain binds an extended helix at the C-terminus of the Dcp2 CD, as in the recent Kl Dcp1-Dcp2-Edc3 structure ${ }^{22}$, and the fully ordered Edc1 activation peptide bridges Dcp1 and Dcp2, similar to the recent $S p$ Edc1-Dcp1-Dcp2 structure ${ }^{19}$. The co-occupancy of Edc1 and Edc3 coactivators in our structure highlights the idea that these decapping factors work together to promote decapping by complementary mechanisms ${ }^{42}$.

Edc1 consolidates the active conformation of Dcp2. Edc1-like coactivator proteins contain tandem, conserved short linear motifs for Dcp1 binding and Dcp2 activation (Fig.1b) $20,21,35,37$. Several structures have now been published that show how the proline-rich Dcp1-binding motif in Edc1-like peptides binds a conserved aromatic cleft on Dcp $1^{19-21,36}$, and our structure displays a very similar interaction between $K l$ Edc1 and $K l$ Dcpl as that observed in $S p, H s$, and chimeric $S p-H s$ structures (Supplementary Fig. 2). The conserved YAGxxF Dcp2-activating motif creates a 3-way interface that bridges Dcp1, Dcp2 NRD, and Dcp2 CD in the catalytically active conformation by threading through a narrow channel between these domains (Fig. 1d,e). In total, the Edc1 peptide interaction with the Dcp1-Dcp2 complex buries $\sim 1300 \AA^{2}$ of surface area, with the YAGxxF Dcp2-activing motif accounting for $\sim 500 \AA^{2}$ of this buried surface. The interface between the Dcp 2 CD and Edc1 peptide covers only $\sim 250 \AA^{2}$ of surface area, consistent with evidence that the peptide alone is insufficient to drive a conformational change in solution ${ }^{19}$.

We have shown that Edcl-like proteins accelerate decapping catalysis by both increasing $k_{\text {cat }}$ and decreasing $K_{\mathrm{M}}{ }^{21,35}$ Kinetic analysis of the Dcp2-activating YAGxxF motif in PNRC2 showed that mutation of the Tyr residue completely abolishes $k_{\text {cat }}$ activation of Dcp2 catalysis, while mutation of Ala and Phe residues significantly impairs activation ${ }^{21}$. We can now rationalize our previous biochemical observations in the context of the fully activated, substrate analog-bound structure. Tyr359 of $K l$ Edc1, the residue most critical for activation, makes a cation $-\pi$ interaction with the strictly conserved Arg39 on the Dcp2 NRD (Fig. 1d). Ala360 packs against conserved hydrophobic residues in the flexible hinge between Dcp2 NRD and CD, and Phe364 plugs into a pocket at the Dcp1-Dcp2 NRD interface consisting of positively charged and hydrophobic residues (Supplementary Fig. 3). These contacts anchor the Edcl activation motif to Dcp1-Dcp2 and provide a complementary surface for the flexible hinge and 130s loop on the Dcp2 CD to pack against Edc1 in the catalytically active conformation of the enzyme. In this way, protein interactions mediated by the Dcp2 activation motif of Edc1 accelerate $k_{\text {cat }}$ not by contacting cap, but instead by limiting torsional degrees of freedom in the hinge to favor the active conformation of Dcp2, in which conserved residues on the CD and NRD recognize and hydrolyze cap using the composite active site.

Residues in Dcp2 NRD and hinge are critical for activation. In order to validate the Edc1-Dcp2 interface observed in our structure and better understand how the Edcl peptide interacts with Dcp2 to promote catalysis, we mutated conserved residues on the Dcp2 NRD, CD, and hinge (Dcp2 residues shown as sticks in Fig. 1d) that contact YAG residues of the Edc1 peptide, and asked how these mutations affect activation of Dcp 2 catalysis by Edc1. Using single-turnover in vitro kinetic decapping assays with cap-radiolabeled RNA substrate and S. pombe Edc1-Dcp1-Dcp2 complex, we find that residues Arg33 ( $K l$ Arg39) and Ile96 ( $K l$ Ile102) are absolutely essential for Edc1 function and that mutation of these conserved residues to Ala or Gly, respectively, completely blocks activation by Edc1 (Fig. 2). In our structure, Arg33 ( $K l$ Arg39) makes a cation- $\pi$ interaction with Edc1 Tyr359 to anchor the activation motif to Dcp2 NRD, and Ile96 ( $K l$ Ile102) 


\section{Table 1 Crystallographic data collection and refinement statistics}

KI Edc1-Dcp1-Dcp2-Edc3 with substrate analog (PDB 6AM0)

\begin{tabular}{ll}
\hline Data collection & \\
\hline Space group & C2 \\
Cell dimensions & \\
\hline$a, b, c(\AA)$ & $174.26,83.25,104.30$ \\
$\alpha, \beta, \gamma\left(^{\circ}\right)$ & $90.00,93.62,90.00$ \\
Resolution $(\AA)$ & $46.0-2.84(3.00-2.84)^{\mathrm{a}}$ \\
$R_{\text {merge }}$ & $0.058(0.78)$ \\
V/ $\sigma$ l & $9.8(1.3)$ \\
$C C_{1 / 2}$ & $99.8(58.5)$ \\
Completeness (\%) & $98.7(99.1)$ \\
Redundancy & $2.9(2.8)$ \\
Refinement & \\
\hline Resolution $(\AA)$ & $46.0-2.84$ \\
No. reflections & 35,286 \\
$R_{\text {work }} / R_{\text {free }}$ & $0.2293 / 0.2491$ \\
No. atoms & \\
\hline Protein & 8610 \\
Ligand & $115^{\mathrm{b}}$ \\
Water & - \\
$B$ factors & \\
\hline Protein & 104 \\
Ligand & $114^{\mathrm{b}}$ \\
Water & - \\
R.m.s. deviations & \\
\hline Bond lengths $(\AA)$ & 0.003 \\
Bond angles $\left({ }^{\circ}\right)$ & 0.665 \\
Ramachandran plot statistics \\
\hline No. favored & $967(95.6 \%)$ \\
No. allowed & $45(4.4 \%)$ \\
No. outliers & $0(0 \%)$ \\
\hline & \\
\hline
\end{tabular}

Data set was collected from a single crystal

Values in parentheses are for highest-resolution shell

b Two molecules of two-headed cap analog and one magnesium ion

packs against Edc1 Ala360 and the Tyr359 backbone. These data suggest that both positioning of the peptide activation motif along the Dcp1-Dcp2 NRD surface, and specific van der Waals interactions with the Dcp2 hinge that links the NRD and CD, are critically important for Edc1-mediated activation.

Interestingly, mutation of other strictly conserved residues on Dcp2 that contact the Edc1 peptide in the catalytically active conformation, such as Phe36 (Kl Phe42) and Tyr92 (Kl Tyr98), have very little effect on Edcl's ability to activate Dcp2. Mutation of Tyr220, a distal residue on the CD that is important for conformational dynamics in Dcp $2^{42}$, also has no effect on activation by Edc1. Importantly, none of the tested mutations to conserved Dcp2 residues resulted in large defects in decapping in the absence of Edc1 (Supplementary Fig. 4), showing that Arg33 (Kl Arg39) and Ile96 (Kl Ile102) are critical specifically for Edc1mediated activation of Dcp2. Finally, in contrast to our previous observations examining Dcp2 activation in the chimeric $H s$ PNRC2-Sp Dcp1-Dcp2 system where we showed PNRC2 peptides with the YAGxxF Dcp2-activating motif had little impact on the $K_{\mathrm{M}}$ of the decapping complex, with native $S p$ Edc1-Dcp1-Dcp2 complexes we see a $\sim 100$-fold decrease in $K_{\mathrm{M}}$ along with a $\sim 10$-fold increase in $k_{\max }$ upon addition of the activator peptide (Supplementary Fig. 5; Supplementary Table 1). This is consistent with recent evidence that $S p$ Edcl peptides can enhance RNA binding to the decapping complex ${ }^{19,20}$.
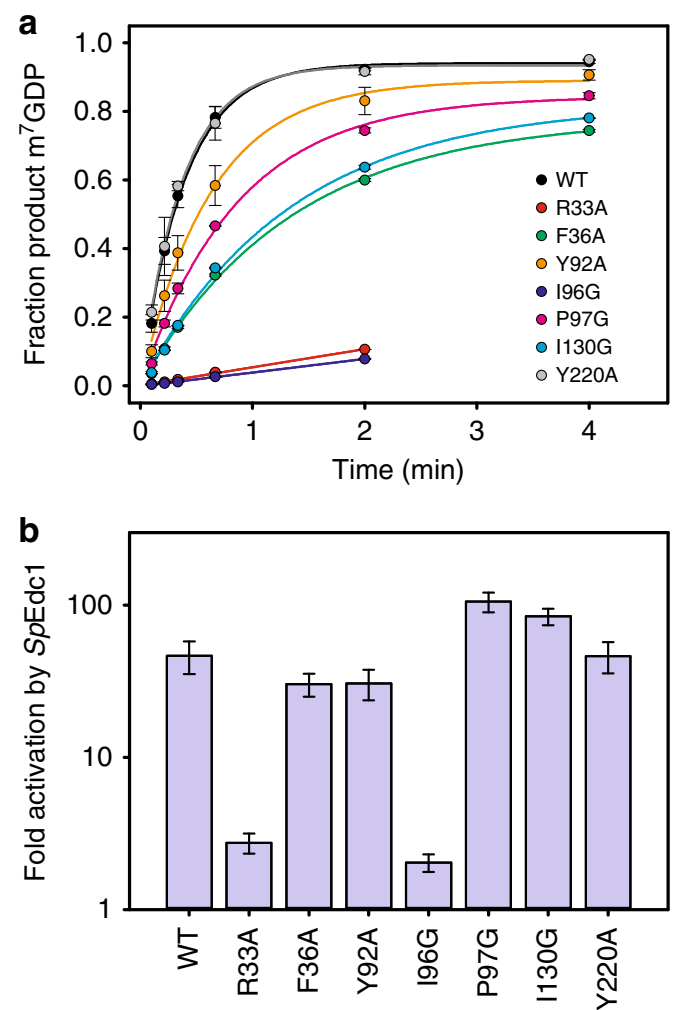

Fig. 2 Mutations on both the Dcp2 NRD and flexible hinge prevent activation by Edc1. a In vitro decapping timecourses for Sp Edc1-Dcp1-Dcp2 WT and Dcp2 mutant complexes. R33A and 196G mutations completely block activation of Dcp1-Dcp2 by Edc1 peptide. Concentration of Dcp1-Dcp2(1-243) is $2 \mu \mathrm{M}$ and Edc1(155-180) peptide is $100 \mu \mathrm{M}$. Errors are s.d. of two independent replicates. b Fold activation of WT and Dcp2 mutant decapping complexes by Edc1 peptide. Calculated as $k_{\text {obs }}\left(\right.$ Edc1-Dcp1-Dcp2)/ $k_{\text {obs }}$ (Dcp1-Dcp2). $k_{\text {obs }}$ values for Dcp1-Dcp2 with no Edc1 coactivator peptide are shown in Supplementary Fig. 4. Errors are s. d. of $k_{\text {obs }}\left(\right.$ Edc1-Dcp1-Dcp2)/ $k_{\text {obs }}($ Dcp1-Dcp2) values obtained from two independent replicates

Substrate analog binds a composite active site on Dcp2. Twoheaded cap substrate analog bound at the Dcp2 active site engages conserved residues on the Dcp2 NRD and CD (Fig. 3a,b). In agreement with our previously reported substrate analog-bound pre-catalytic structure ${ }^{21}$, and recently reported $\mathrm{m}^{7} \mathrm{GDP}$ productbound structures ${ }^{19,22}$, the $\mathrm{m}^{7} \mathrm{G}$ of cap is recognized by conserved, catalytically critical Trp and Asp residues on the Dcp2 NRD. The opposite face of this $\mathrm{m}^{7} \mathrm{G}$ nucleotide packs against the backbone of the Dcp2 CD 190s loop in the catalytically active conformation. This agrees with recent product-bound structures, but differs from the pre-catalytic conformation, in which this face of $\mathrm{m}^{7} \mathrm{G}$ was engaged in base stacking with Sp Tyr220 ( $K l$ Phe223). In the active conformation reported here however, the $\mathrm{CD}$ is rotated $\sim 90^{\circ}$ from the pre-catalytic conformation, and Phe223 base stacks with the second $\mathrm{m}^{7} \mathrm{G}$ of our two-headed substrate analog, likely mimicking binding of the first transcribed nucleotide of RNA substrate to the Dcp 2 CD. Additional conserved and catalytically important residues make contacts with the substrate analog phosphate chain, including the general acid Lys134 and Arg132 on the CD, and Lys99 on the NRD.

Substrate analog positioning suggests a path for RNA binding. Our structure suggests that the first transcribed nucleotide of RNA binds Phe223 at the top of an extended, positively charged 
a

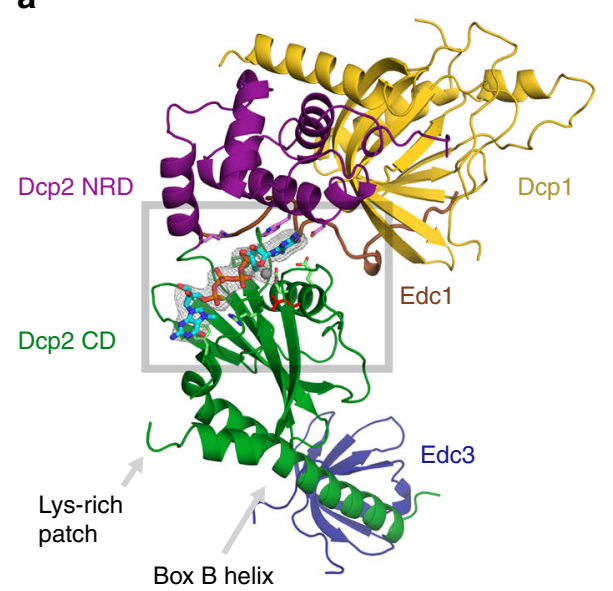

b

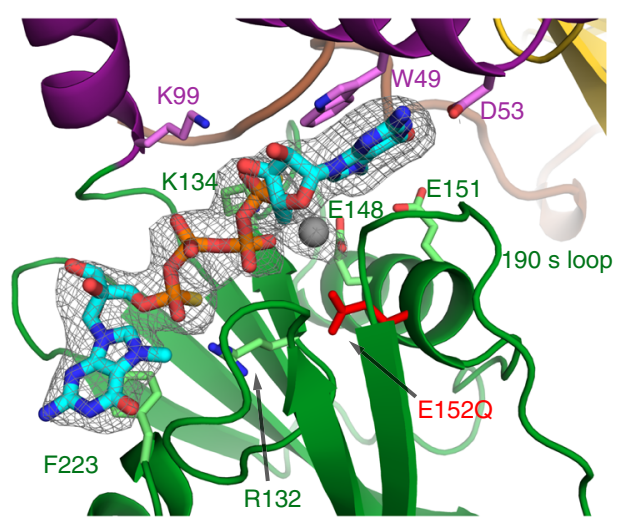

C

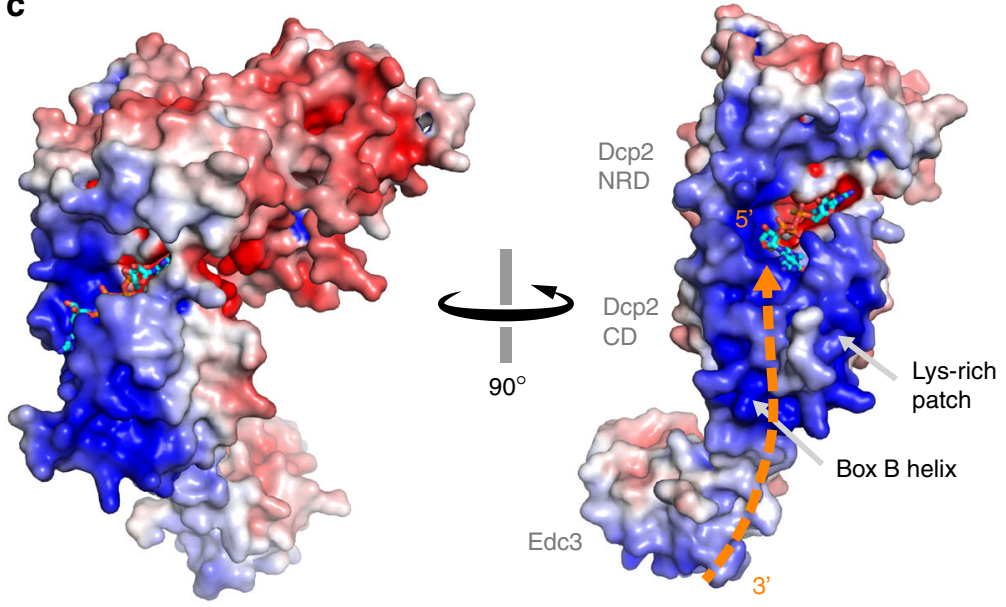

d

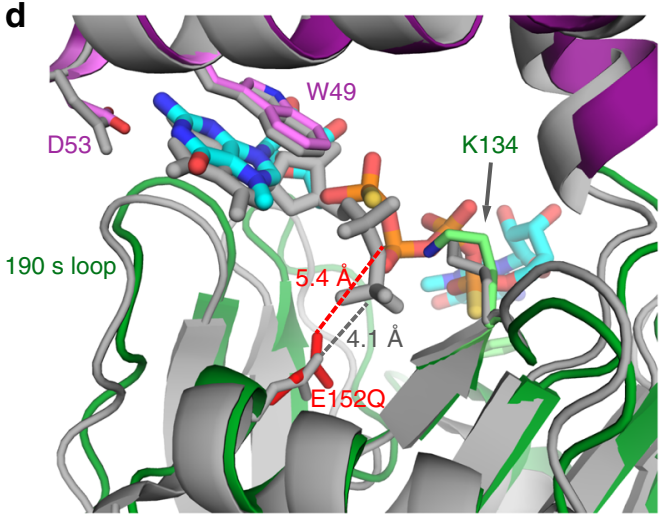

Fig. 3 Substrate analog binding to the active conformation of Edc1-Dcp1-Dcp2-Edc3 decapping complex. a Full Edc1-Dcp1-Dcp2-Edc3 structure showing substrate analog binding between the Dcp2 NRD and CD. b Close-up of substrate analog bound at the Dcp2 active site. Conserved, catalytically important residues that contact the cap analog are shown as sticks. The catalytic Glu base (mutated to Gln in our structure) is colored red; $\mathrm{Mg}^{2+}$ ion is shown as a gray sphere; $F_{\mathrm{o}}-F_{\mathrm{c}}$ omit map for cap analog and $\mathrm{Mg}^{2+}$ is shown at $2.5 \sigma$ in gray. c Edc1-Dcp1-Dcp2-Edc3 complex with calculated electrostatic surface ( 6 to $+6 \mathrm{kT} / \mathrm{e}$ ) in the same orientation as in (a) (left), and rotated $90^{\circ}$ (right). Dotted orange line shows the predicted path of RNA based on the orientation of substrate analog in the active site of Dcp2. The 5' cap of RNA binds in the Dcp2 composite active site (substrate analog in cyan), RNA follows the positively charged Dcp2 CD C-terminal Box B helix past a conserved Lys-rich patch, to Edc3 coactivator. d Alignment of the substrate analog-bound (colored structure, PDB 6AM0) and product-bound (gray, PDB 5LOP ${ }^{22}$ ) K. lactis Dcp2 structures; view is rotated $180^{\circ}$ from that shown in (a) and (b). The Dcp2 NRD (residues 8-97) was used for alignment, giving a backbone RMSD of $0.78 \AA$; all-atom RMSD for alignment of both the NRD and CD (residues 8-240) of substrate analog versus product-bound Dcp2 is $1.6 \AA$. Distance from catalytic Glu residue to $\beta$-phosphate of substrate analog or product are shown as red and gray dotted lines, respectively

C-terminal helix on the CD of Dcp2 (Box B helix, Fig. 3a). Based on this cap orientation, the RNA body likely extends down the positively charged Box B helix, past a conserved Lys-rich patch on the dorsal surface of Dcp2, to coactivator Edc3. This predicted RNA-binding path is consistent with many lines of biophysical and biochemical evidence, including: (1) NMR chemical shift perturbations induced by RNA binding on the surface of the Dcp2 $\mathrm{CD}^{19,26}$, (2) biochemical data showing that the Box B helix is critical for decapping activity in vitro 25 , (3) kinetic analysis showing that mutations to basic residues in both the Box B helix and Lys-rich patch significantly increase substrate $K_{\mathrm{M}}{ }^{26}$, (4) SPR data showing that mutations in the Box B helix significantly weaken RNA binding ${ }^{17}$, (5) yeast growth assays showing that mutations in the Box B helix and Lys-rich patch result in temperature-sensitive growth defects ${ }^{26}$, and (6) evidence that Edc3 activates decapping in part by enhancing RNA binding to the decapping complex ${ }^{22}$. The positioning of substrate analog in the Dcp2 active site revealed by our Edc1-Dcp1-Dcp2-Edc3 structure suggests an RNA-binding path along the Dcp2 Box B helix that nicely agrees with all of this previous RNA binding and activity data, adding structural support to the RNA-binding path that has been hypothesized for some time by ourselves and others.

Substrate and product-bound Dcp2 conformations are similar. Alignment of the Dcp2 catalytic core from the active conformation substrate analog and product-bound structures shows that these adopt overall very similar Dcp2 domain orientations (Fig. 3d; Supplementary Fig. 1a). This is consistent with NMR chemical shift data that suggest the Edc1-Dcp1-Dcp2 complex with either product $\mathrm{m}^{7} \mathrm{GDP}$ or capped RNA substrate have similar conformations in solution ${ }^{19}$. The most notable difference in the aligned substrate analog and product-bound Dcp2 structures is the difference in positioning of the phosphate chain of the cap. In the substrate analog-bound state, the $\beta$ phosphate, which undergoes nucleophilic attack by water during cap cleavage, is located $5.4 \AA$ from the general base Glu152. This is very similar to the substrate-Glu distance in the prototypical Nudix hydrolase MutT, in which a solution-state NMR structure showed the catalytic Glu residue to be $\sim 6 \AA$ from the nonhydrolyzable AMPCPP substrate, with an intervening water molecule well-positioned for nucleophilic attack of the $\beta$ - 
phosphate ${ }^{51}$. In the post-catalytic, $\mathrm{m}^{7} \mathrm{GDP}$ product-bound structure, the $\beta$-phosphate of cap is positioned nearer to the Nudix helix, at a distance of $4.1 \AA$ from the general base Glu152.

Substrate and product-bound structures also differ in the number of magnesium atoms located at the active site. In our substrate analog-bound structure, we were able to build one magnesium atom that is coordinated by Glu148. Although there is some additional density present in the $F_{\mathrm{o}}-F_{\mathrm{c}}$ difference map at the active site (Supplementary Fig. 6a), this was too weak to reliably build additional $\mathrm{Mg}^{2+}$ or water. This agrees well with a high-resolution structure of the $S$. cerevisiae Dcp2 CD, which showed a single magnesium atom coordinated directly to $\mathrm{Sc}$ Glu149 ( $K l$ Glu148), which is coordinated by other conserved Glu residues through water-mediated contacts. In both the K. lactis and $S$. pombe product-bound structures, however, three magnesium atoms are placed in the Dcp2 active site, coordinated by the conserved CD Glu residues and the phosphate groups of $\mathrm{m}^{7} \mathrm{GDP}$ (Supplementary Fig. 6b,c). Enzymology from our lab has shown that the $\mathrm{Mg}^{2+}$-dependence of decapping kinetics fits a hill coefficient of 2.4, suggesting Dcp2 binds at least two metal ions ${ }^{52}$, which is most consistent with the product-bound structures. In the end, both our substrate analog-bound structure $(2.84 \AA$ resolution) and the product-bound structures ( $3.1 \AA$ and $3.5 \AA$ for $\mathrm{PDB} 5 \mathrm{~N} 2 \mathrm{~V}^{37}$ and $5 \mathrm{LOP}^{22}$, respectively) are not at sufficient resolution to very accurately distinguish $\mathrm{Mg}^{2+}$ and water molecules, and high-resolution structures of Dcp2 with bound substrate and product will be required to unambiguously determine the location and precise role of coordinated magnesium and water molecules in the active site.

Dcp2 shows selectivity for the first transcribed nucleotide. As discussed above, our structure of substrate analog-bound Dcp2 suggests that the first transcribed nucleotide of RNA is recognized by the conserved aromatic Phe223 (Sp Tyr220). Surprisingly, although mutation of this residue produces temperature-sensitive growth defects in yeast ${ }^{26}$, Tyr220Ala mutations in our single-turnover in vitro decapping assays with $S$. pombe Edc1-Dcp1-Dcp2 or Dcp1-Dcp2 complex had no effect on catalysis (Fig. 2a,b; Supplementary Fig. 4). In the apo, inactive conformation of Dcp1-Dcp2 that predominates in solution ${ }^{19}$, Tyr220 ( $K l$ Phe223) interacts with conserved aromatic W43 on the NRD of Dcp2 (e.g., PDB $2 \mathrm{QKM}^{17}, 5 \mathrm{~J}^{20} \mathrm{~K}^{20} 5 \mathrm{KQ} 1^{21}$ ), which likely stabilizes the inactive conformation of the enzyme. Furthermore, we have recently shown that Tyr220 plays an important role in mediating Dcp2 conformational dynamics and that mutation of this residue alleviates an autoinhibited conformation of the apo enzyme ${ }^{42}$. It is therefore likely that Tyr220 is a multifunctional residue, which stabilizes an inactive/autoinhibited conformation of Dcp2 in the apo state and acts as a nucleotide binding platform in the substrate-bound, active conformation. In this way, mutation of Tyr220 may both accelerate catalysis by alleviating autoinhibition and impair catalysis by causing defects in substrate binding, resulting in decapping kinetics that appear relatively unaffected by Tyr220Ala mutation.

It was recently reported that Dcp2-mediated decapping in mammals is inhibited by $\mathrm{m}^{6} \mathrm{~A}$ modification of the first transcribed nucleotide of RNA substrates ${ }^{15}$, suggesting that Dcp2 may be selective for the identity of the first transcribed nucleotide, as is observed in the bacterial decapping enzyme $\mathrm{NudC}^{53}$. Accordingly, we next asked if Dcp2 selectivity for the first transcribed nucleotide of RNA substrate depends on Tyr220. We compared decapping kinetics for cap-radiolabeled 29-mer RNA substrates where the first transcribed nucleotide was either guanosine (G-RNA), adenosine (A-RNA), or cytosine (C-RNA) using WT, or Tyr220Gly Sp Edc1-Dcp1-Dcp2 decapping
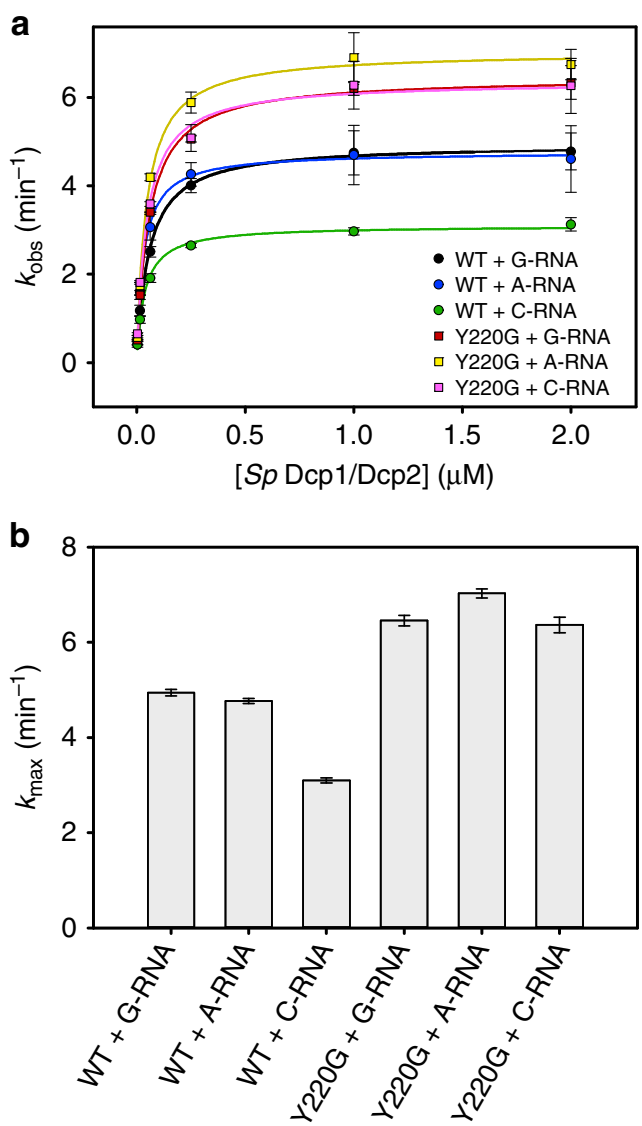

Fig. 4 Dcp2 selectivity for the first transcribed nucleotide of RNA depends on Tyr220 (KI Phe223). a Plots of $k_{\text {obs }}$ for decapping a 29-mer RNA substrate with $\mathrm{G}, \mathrm{A}$, or $\mathrm{C}$ as the first transcribed nucleotide (G-RNA, ARNA, C-RNA, respectively), with WT or Tyr220Gly S. pombe

Edc1-Dcp1-Dcp2 complex versus enzyme concentration. The concentration of the Dcp1-Dcp2(1-243) complex is varied, with Edc1(155-180)

coactivator peptide kept at saturating concentration $(50 \mu \mathrm{M})$. Errors on $k_{\mathrm{obs}}$ are s.d. of two independent replicates. b Bar graph showing $k_{\max }$ values determined from $k_{\text {obs }}$ versus enzyme concentration plots in (a). Errors are standard error of the fits to determine $k_{\max }$

complexes (Fig. 4; Supplementary Table 2). Tyr220Gly mutations were used here to ensure maximal disruption of hydrophobic stacking interactions with substrate; this mutation also resulted in a small acceleration of decapping, likely resulting from disruption of the Y220-W43 interaction in the inactive Dcp2 conformation discussed above. With WT decapping complex, $k_{\max }$ for both GRNA and A-RNA is $~ 2$-fold faster than C-RNA. Upon mutation of Tyr220, decapping is accelerated and the selectivity of $5^{\prime}$ G/A versus $\mathrm{C}$ nucleotides is abolished, resulting in approximately the same $k_{\max }$ value for G-RNA, A-RNA, and C-RNA. This suggests that Dcp2 discriminates between G/A versus $C$ at the first transcribed nucleotide position during the catalytic step of decapping, and that this selectivity critically depends on Tyr220 (Kl Phe223). Dcp2's Tyr220-dependent preference for G/A versus $\mathrm{C}$ at this position is in line with the observation that there is a strong genome-wide bias, in both fission yeast ${ }^{54}$ and humans ${ }^{55}$, toward purines $(\mathrm{G}, \mathrm{A})$ over pyrimidines $(\mathrm{C}, \mathrm{U})$ at the transcription start site +1 position. Furthermore, our selectivity data supports the hypothesis that Tyr220 ( $K l$ Phe223) is important for recognition of the first transcribed nucleotide and that the apparent lack of kinetic defects, or even acceleration of decapping, when this residue is mutated may arise from its 
multi-functional role in different conformations of the enzyme ${ }^{19,42}$.

Finally, we also asked whether the activated $S$. pombe decapping complex showed selectivity for $\mathrm{A}$ versus $\mathrm{m}^{6} \mathrm{~A}$ at the first transcribed nucleotide position of RNA, as has been recently shown for mammalian Dcp2 $2^{15}$. In contrast to the mammalian decapping enzyme, we found that fission yeast Edc1-Dcp1-Dcp2 complex, or Dcp2 alone, showed no selectivity for A versus $\mathrm{m}^{6} \mathrm{~A}$ at the $5^{\prime}$-most nucleotide position on a 29-mer RNA substrate in in vitro decapping assays (Supplementary Fig. 7). This suggests that recognition of the $\mathrm{m}^{6} \mathrm{~A}$ modification at the first transcribed nucleotide of mRNA $\left(\mathrm{m}^{6} \mathrm{~A}_{\mathrm{m}}\right.$ in mammals, where this nucleotide is also 2'-O-methylated) by Dcp2 may only be conserved in higher eukaryotes.

\section{Discussion}

Several recent studies have provided new structural information about the mRNA-decapping enzyme Dcp $2^{19-22}$. These include structures of the Dcp1-Dcp2 heterodimer with markedly different conformations of Dcp2, bound cap analog or $\mathrm{m}^{7} \mathrm{GDP}$ product, and in complex with Edc1-like or Edc3 coactivators (Fig. 5a). Here we expand on the structural understanding of Dcp2 activation and catalysis with a new structure of the fourprotein complex Edc1-Dcp1-Dcp2-Edc3 in the catalytically active conformation of Dcp2 with a tight-binding substrate ana$\log$ bound to the enzyme active site (Fig. 1a-c, Table 1). The coactivator Edc1 peptide engages conserved residues on Dcp1-Dcp2 NRD, providing a complementary surface against which the Dcp2 CD and flexible hinge pack in the catalytically active conformation, thus consolidating this conformation and promoting catalysis (Fig. 1d,e and 2) Cap substrate analog binds to the enzyme active site in an orientation that positions the RNA body to follow an extended, positively charged helix of Dcp2 to the bound Edc3 coactivator (Fig. 3), which has been shown to enhance RNA binding to these complexes in vitro ${ }^{22}$. Finally, we show that the conserved aromatic residue Tyr220 ( $K l$ Phe223) that binds the second nucleotide of our substrate analog is critical for recognition of the first transcribed nucleotide in RNA by the decapping complex (Fig. 4). Purine specificity is conferred for this nucleotide during the catalytic step of decapping.

Many different conformations of the decapping enzyme Dcp2 have been structurally characterized, but a consensus is now emerging around the identity of the catalytically active conformation of Dcp2. With the addition of the Edc1-Dcp1-Dcp2-Edc3 cap analog structure presented here, we now have both product $\mathrm{m}^{7} \mathrm{GDP}$ and substrate analog-bound structures of the Dcp1-Dcp2 decapping complex in what can safely be described as the active conformation (Fig.5a, active structures). The substrate and product-bound active conformations are nearly identical, with major differences only in the positioning of the cap phosphate chain before and after hydrolysis (Fig. 3d). These structures all show that the $\mathrm{m}^{7} \mathrm{G}$ nucleotide of cap is specifically recognized by stacking with a conserved Trp and hydrogen bonding to a conserved Asp residue on the Dcp2 $\mathrm{NRD}$, explaining why the NRD contributes 100 -fold to catalysis $^{31}$. In the active conformation, the 190s loop of the Dcp2 CD packs against the opposite face of the $m^{7} \mathrm{G}$ base, which brings the Nudix helix and catalytic Glu within $\sim 6 \AA$ of the cap $\beta$-phosphate, close enough to carry out hydrolysis chemistry and cap cleavage (Fig. 3b).

The catalytic importance and roles of the additional conformations of Dcp2 that have been structurally characterized, but are very different from the active conformation (Fig. 5a, inactive, RNA-binding, and pre-catalytic structures; Fig. 5b,c), remain less clear. In our previously published pre-catalytic substrate analog- bound PNRC2-Dcp1-Dcp2 structure ${ }^{21}$, Dcp2 adopts a conformation in which the NRD recognizes one $\mathrm{m}^{7} \mathrm{G}$ nucleotide of cap analog as in the active conformation, but the CD of Dcp2 is rotated $90^{\circ}$ and $S p$ Tyr220 stacks against the opposite face of this $\mathrm{m}^{7} \mathrm{G}$, rather than the 190s loop (Fig. 5a, pre-catalytic; Fig. 5c). In the pre-catalytic conformation, the second $\mathrm{m}^{7} \mathrm{G}$ nucleotide of our two-headed cap analog is bound by the strictly conserved $S p$ Tyr92 residue near the Dcp2 hinge. In the Edc1-bound active conformation, however, this Tyr is occluded by interaction with the Dcp2-activating motif on Edc1 (Fig. 5d). It is possible that this pre-catalytic conformation, in which cap analog is bound by a composite nucleotide binding site consisting of conserved residues on the Dcp2 CD and NRD, represents an early step in cap recognition that precedes formation of the active conformation and its consolidation by the Edc1 YAGxxF motif. Likewise, the apo Edc1-Dcp1-Dcp2 structure by Valkov et al. ${ }^{20}$, which adopts a conformation that positions RNA-binding residues on the Dcp2 CD and Dcp1 opposite each other to create a positively charged channel, may represent a conformation of the apo enzyme that is primed for RNA binding or scanning ${ }^{21,24}$ (Fig. 5a; RNA-binding). With both of these alternative conformations, however, it is so far difficult to rule out the possibility that these are simply offpathway conformations captured by crystallization. This is not to say that valuable information is not provided by conserved cap or coactivator contacts with the enzyme, but rather that these specific conformations may not be on-pathway in the Dcp2 catalytic cycle. Indeed, recent PRE NMR data suggests that the apo Edc1bound conformation in the Valkov et al. structure is not populated in solution in vitro ${ }^{19}$. In these same experiments, it was not possible to distinguish our pre-catalytic substrate-bound conformation from the closed, inactive conformation of Dcp2 that predominates in solution (Fig. 5a, inactive; Fig. 5b).

Building on recent reports of Dcp2 autoinhibition in yeast ${ }^{29}$, our lab has now shown that the C-terminal extension in fission yeast Dcp2 contains autoinhibitory motifs that interact with the core domains of Dcp1-Dcp2 to inhibit the decapping complex and impair activation by Edcl, and that this autoinhibition is alleviated by coactivator Edc3 ${ }^{42}$. The Edc1-Dcp1-Dcp2-Edc3 structure presented here provides the structural framework to begin to understand how coactivators act to alleviate autoinhibition and activate catalysis. We suggest a model of decapping regulation whereby full-length Dcp2 is autoinhibited and coactivators Edc3 and Edc1 act simultaneously to (1) alleviate this autoinhibition, and (2) activate decapping catalysis by both consolidating the Dcp2 active conformation and by enhancing RNA binding to the complex. In this way, switchlike mutli-log unit changes in decapping activity are easily achievable when multiple coactivators bind Dcp2 and function simultaneously. Because many decapping coactivators have genetic or physical interactions with one another $29,45-49$, we anticipate that combinatorial control of decapping via multiple coactivators is a general and important principle for regulation of mRNA decay.

\section{Methods}

Protein expression and purification. K. lactis his-tev-Dcp1(1-188)-Dcp2(1-275, E152Q)-Edc3(1-66) polycistronic construct used for crystallization experiments was obtained as a single $E$. coli codon-optimized DNA sequence with a T7 promoter preceding each protein as a gBlock from Integrated DNA Technologies (his is hexahistadine affinity tag, tev is Tobacco Etch Virus protease cleavage site; see Supplementary Table 3 for sequence). This was cloned into a Kanamycin-resistant expression vector and transformed into E. coli BL21-star DE3 cells (Invitrogen; see Supplementary Table 4 for primers used). Cells were grown in LB media to OD $\sim 0.6$, protein expression was induced with IPTG for $18 \mathrm{~h}$ at $20^{\circ} \mathrm{C}$. Cells were harvested at $5000 \times g$, lysed by sonication, and clarified at $16,000 \times g$ in lysis buffer (50 mM sodium phosphate $\mathrm{pH} 7.5,300 \mathrm{mM}$ sodium chloride, $10 \mathrm{mM}$ imidazole, $5 \%$ glycerol, $10 \mathrm{mM}$ 2-mercaptoethanol, Roche EDTA-free protease inhibitor cocktail). The protein complex was purified by Ni-NTA affinity chromatography 
a

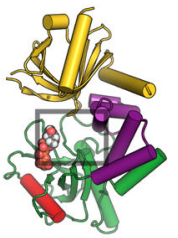

Nucleotides

ATP

Coactivators

Conformation

Organism

PDB ID

Citation

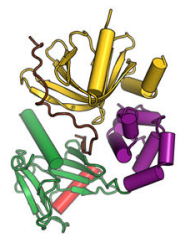

Edc1

RNA-binding?

S. pombe

5J3T

Valkov et al. 2016

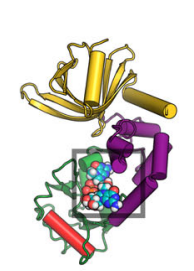

Cap substrate analog

Pre-catalytic?

S. pombe

5KQ4

Mugridge et al.

2016

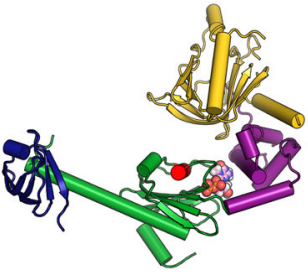

m7GDP product

Edc3

Active

K. lactis

5LOP

Charenton et al. 2016

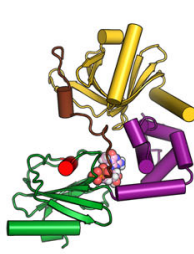

m7GDP product Edc1

Active

S. pombe

$5 \mathrm{~N} 2 \mathrm{~V}$

Wurm et al. 2017

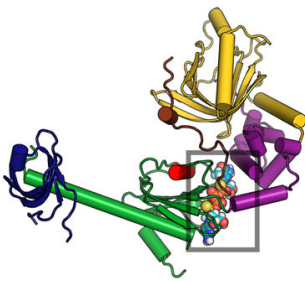

Cap substrate analog

Edc1

Edc3

Active

K. lactis

6AM0

Mugridge et al.

2018

\section{b}

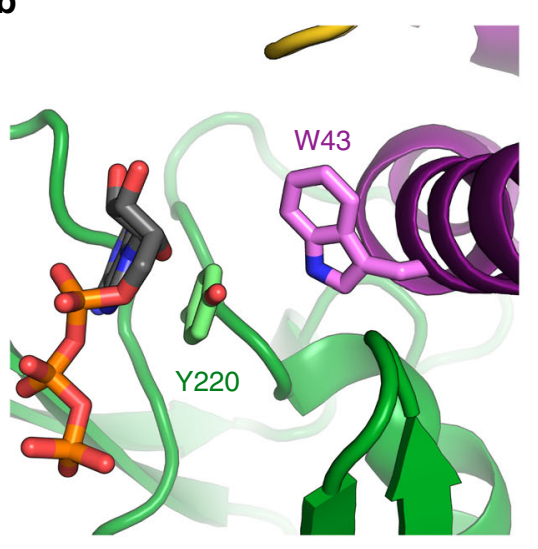

C

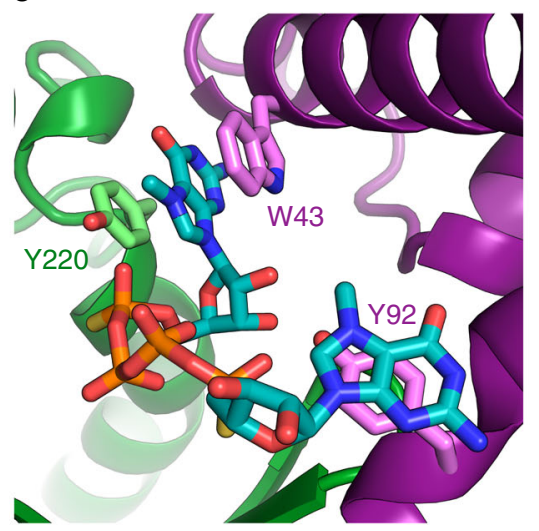

d

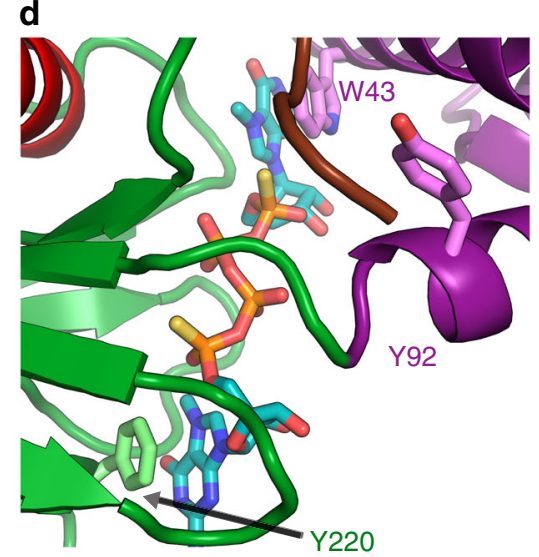

Fig. 5 Summary of recent Dcp1-Dcp2 structures and interactions between important aromatic cap-binding residues in different conformations. a Gallery showing the different overall conformations of recent Dcp1-Dcp2 structures. Text lists bound nucleotides, coactivators, and proposed description of the Dcp2 conformation. Dcp1 is yellow, Dcp2 NRD is purple, Dcp2 CD is green with the catalytic Nudix helix colored red, Edc1 is brown, Edc3 is dark blue, cap substrate analog is cyan, $\mathrm{m}^{7} \mathrm{GDP}$ product is pink. From left to right, PDB codes and citations are $2 \mathrm{QKM}{ }^{17}, 5 \mathrm{~J} 3 \mathrm{~T}^{20}, 5 \mathrm{KQ}^{21}, 5 \mathrm{LOP} 22,5 \mathrm{~N}^{2} \mathrm{~V}^{19}, 6 \mathrm{AMO}$ (this work). b Close-up view of ATP-bound inactive structure (PDB 2QKM) ${ }^{17}$. Conserved, cap-binding residues Trp43 and Tyr220 make edge-on contacts in this inactive conformation. ATP is gray. c Close-up view of cap analog-bound, pre-catalytic structure (PDB 5KQ4) ${ }^{21}$. Residues Tyr220 and Trp43 bind one $\mathrm{m}^{7} \mathrm{G}$ nucleotide of two-headed cap analog, Tyr92 binds the second $\mathrm{m}^{7} \mathrm{G}$ nucleotide of the cap analog. $\mathbf{d}$ Close-up view of cap analog-bound, active structure (PDB 6AM0); S. pombe residue numbering is used here instead of $K$. lactis for comparison with structures shown in (b) and (c). Trp43 (KI Trp49) binds one $\mathrm{m}^{7} \mathrm{G}$ nucleotide of two-headed cap analog and Tyr220 (KI Phe223) binds the second $\mathrm{m}^{7} \mathrm{G}$ of cap analog, mimicking binding of the first transcribed nucleotide. Tyr92 (KI Tyr98) is buried in a protein-protein interface with Edc1 coactivator in the active conformation

and solubility/affinity tags were cleaved by treatment with TEV protease overnight at room temperature in elution buffer $(50 \mathrm{mM}$ sodium phosphate $\mathrm{pH} 7.5,300 \mathrm{mM}$ sodium chloride, $250 \mathrm{mM}$ imidazole, $10 \mathrm{mM}$ 2-mercaptoethanol). The $\mathrm{Kl}$

Dcp1-Dcp2-Edc3 complex was separated from cleaved tags by size exclusion chromatography on a GE Superdex $20016 / 60$ gel filtration column in crystallization buffer (10 mM HEPES $\mathrm{pH} 7,150 \mathrm{mM} \mathrm{NaCl}, 1 \mathrm{mM}$ DTT). The purified complex was concentrated to $15 \mathrm{mg} / \mathrm{mL}$ and flash frozen in liquid nitrogen for crystallization experiments.

S. pombe his-GB1-tev-Dcp1(1-127)-Dcp2(1-243) constructs used for kinetic experiments were obtained by cloning the genomic Sp Dcp2(1-243) coding sequence into MCS2 of Novagen pACYC co-expression vector, and an E. Coli codon-optimized $S p$ Dcp1(1-127) DNA sequence obtained from Integrated DNA Technologies into MCS1 of the PACYC vector containing an N-terminal his-GB1tev tag (GB1 is a solubility tag derived from the B1 domain from Protein G; see Supplementary Table 3 for codon-optimized $S p$ Dcpl sequence). The $S p$ Dcp1-Dcp2 protein complex was expressed and purified as described above for the $K l$ Dcp1-Dcp2-Edc3 complex. After TEV cleavage, the complex was separated from cleaved tags by size exclusion chromatography on a GE Superdex 200 16/60 gel filtration column in storage buffer (50 mM HEPES pH 7.5, $100 \mathrm{mM} \mathrm{NaCl}, 5$ mM DTT). The purified $S p$ Dcp1-Dcp2 complex was concentrated, glycerol added to a final concentration of $20 \%$, and flash frozen in liquid nitrogen; final concentration was $200 \mu \mathrm{M}$. Point mutants of the Sp Dcp1-Dcp2 complex were made by whole plasmid PCR with mutagenic divergent primers, verified by sequencing, and purified as above (see Supplementary Table 4 for primer sequences).

Crystallization of Edc1-Dcp1-Dcp2-Edc3 with substrate analog. $K l$ Edc1(355380) peptide (from gene KLLA0_A01474g) was chemically synthesized by Peptide 2.0 and dissolved in crystallization buffer to a concentration of $5 \mathrm{mM}$. Two-headed cap substrate analog, $\mathrm{m}^{7} \mathrm{Gp}(\mathrm{S}) \mathrm{ppp}(\mathrm{S}) \mathrm{m}^{7} \mathrm{G}^{50,56}$, was dissolved in water at a concentration of $100 \mathrm{mM}$ and the $\mathrm{pH}$ was adjusted to $\sim 7$.

Protein solution consisting of $4 \mathrm{mg} / \mathrm{mL}$ purified K. lactis Dcp1(1-188)-Dcp2 (1-275, E152Q)-Edc3(1-66), $1 \mathrm{mM} \mathrm{Kl} \mathrm{Edc1} \mathrm{peptide,} 6 \mathrm{mM}$ substrate analog, and $10 \mathrm{mM} \mathrm{MgCl}_{2}$ was prepared in crystallization buffer and incubated at room temperature for $30 \mathrm{~min}$. Using a TTP Labtech Mosquito Crystal robot, $250 \mathrm{~nL}$ of protein solution was combined with $250 \mathrm{~nL}$ of well solution, and then $50 \mathrm{~nL}$ of seed stock, in a hanging-drop vapor diffusion experiment. Crystallization drops were prepared at room temperature and immediately moved to $4{ }^{\circ} \mathrm{C}$. The well solution contained $220 \mathrm{mM}$ magnesium acetate, $30 \mathrm{mM}$ EDTA, and $8 \% \mathrm{w} / \mathrm{v}$ PEG 8000 . The seed stock was prepared using Hampton's Seed Bead kit, using crystals grown with 1:1 protein solution and well solution containing $200 \mathrm{mM}$ magnesium acetate and $10 \% \mathrm{w} / \mathrm{v}$ PEG 8000 at room temperature, diluted 10,000-fold. Block-shaped crystals $\sim 40-80 \mu \mathrm{m}$ in size grew at $4{ }^{\circ} \mathrm{C}$ within 1-2 days. Crystals were flash frozen in liquid nitrogen using a cryoprotectant consisting of well solution with 15\% PEG 8000. 
Crystallographic data collection and refinement. All data were collected at Lawrence Berkeley National Lab, Beamline 8.3.1 at the Advanced Light Source on a Dectris Pilatus3 S 6 M detector at $100 \mathrm{~K}$ and a wavelength of $1.11583 \AA$ A. Data were indexed, integrated and scaled using $\mathrm{XDS}^{57}$. The structures were solved in space group $\mathrm{C} 2$ by molecular replacement using Phaser in the CCP4 suite ${ }^{58}$. Fragments of chains A, B, and C of PDB 5LOP were used as molecular replacement models ${ }^{22}$, first placing Dcp1-Dcp2 NRD(1-96), then Dcp2 CD(104-240), then Dcp2 (252-260)-Edc3(1-66). The asymmetric unit contained two nearly symmetric copies of the Edc1-Dcp1-Dcp2-Edc3 complex. In the crystal lattice, one molecule of Edc3 Lsm domain forms a dimer as in the $K l$ Edc3-Dcp1-Dcp2-m ${ }^{7}$ GDP product structure (PDB 5LOP) ${ }^{22}$, but the spacegroups $\left(\mathrm{C}_{2}\right.$ for $\mathrm{AM} 60$ and $\mathrm{P}_{4} 22$ for $5 \mathrm{LOP}$ ) and overall crystal packing are entirely different between our structure and the previously determined $K$. lactis structure. Molecular replacement failed to correctly place the Dcp2(252-260)-Edc3(1-66) fragment for chain $\mathrm{H}$, as the density was very weak for this chain of Edc3, likely because this molecule of Edc3 does not form a dimer in the crystal lattice. This fragment was placed manually in COOT, based on strong density for the Dcp2(252-260) Edc3-binding motif and strong density for some $\beta$-strands in Edc3. After a preliminary round of refinement including rigid-body refinement in PHENIX ${ }^{59}$, the Edc1 peptide, Dcp2 hinge, and Dcp2 extended helix were built manually in $\mathrm{COOT}^{60}$. The structure was then iteratively refined in PHENIX and manually adjusted in COOT. Final refinement used Non-Crystallographic Symmetry, Translation-Libration-Screw parameterization for chain $\mathrm{H}$, and secondary structure, stereochemical and atomic displacement parameter restraints. For the final model of $K l$ Edc1-Dcp1-Dcp2-Edc3, all residues were in favored (95.6\%) or allowed (4.4\%) regions of the Ramachandran plot as evaluated by MolProbity ${ }^{61}$.

Structural analysis and visualization. All alignments and structural figures were prepared using PyMol version 1.7. Electrostatic surfaces were calculated using PDB2PQR \& APBS webservers (http://nbcr-222.ucsd.edu/pdb2pqr_2.0.0/) ${ }^{62,63}$ using the AMBER94 forcefield to calculate charges. Electrostatic surfaces were visualized in PyMol using APBS tools 2.1. Buried surface area in protein interfaces was calculated using the PDBePISA webserver (http://www.ebi.ac.uk/pdbe/ prot_int/pistart.html) ${ }^{64}$.

Kinetic decapping assays. For the decapping assays measuring $k_{\mathrm{obs}}$ for Dcp1-Dcp2 point mutants \pm Edcl described in Fig. 2 and Supplementary Fig. 4, SP Dcp1-Dcp2(1-243) WT and mutant complexes had a final concentration of $2 \mu \mathrm{M}$ and $S p$ Edc1 (155-180) peptide had a final concentration of $100 \mu \mathrm{M}$. Sp Edcl (155-180) peptide was chemically synthesized by Peptide 2.0 and stock solutions were prepared at $5 \mathrm{mM}$ concentration in crystallization buffer, flash frozen in liquid nitrogen, and stored at $-20^{\circ} \mathrm{C}$. These experiments used an in vitro transcribed, enzymatically capped 355-mer RNA substrate derived from MFA2 and ${ }^{32} \mathrm{P}$-labeled at the $\alpha$-phosphate of cap. Reactions were carried out in the decapping buffer used previously to measure PNRC2 activation kinetics $(50 \mathrm{mM}$ HEPES pH 7, $100 \mathrm{mM}$ $\mathrm{KCl}, 10 \%$ glycerol, $5 \mathrm{mM} \mathrm{MgCl}$, with RNase inhibitor and $0.1 \mathrm{mg} / \mathrm{mL}$ acetylated BSA $)^{21} .3 \times$ protein solutions with Dcp1-Dcp2+Edc1 peptide, and $1.5 \times$ capped RNA solutions were equilibrated separately at $4{ }^{\circ} \mathrm{C}$ for $30 \mathrm{~min}$ prior to initiation. Reactions were initiated by combining $15 \mu \mathrm{L} 3 \times$ protein solution with $30 \mu \mathrm{L} 1.5 \times$ RNA solution at $4{ }^{\circ} \mathrm{C}$. Time points were quenched by the addition of excess EDTA, TLC was used to separate RNA from product $\mathrm{m}^{7} \mathrm{GDP}$, and the decapped fraction was quantified using a GE Typhoon scanner and ImageQuant software. Plots of fraction $\mathrm{m}^{7} \mathrm{GDP}$ product versus time were fit to a first order exponential to obtain $k_{\text {obs; }}$ in the case of R33A and I96G point mutants, decapping kinetics were too slow to obtain reliable exponential fits and $k_{\text {obs }}$ was obtained from a linear fit of initial rates (less than $20 \%$ decapped), by dividing the slope by the average endpoint of 0.95. Errors on individual time points in Fig. 2a are the s.d. between two independent repetitions of the decapping reaction; errors on $k_{\mathrm{obs}}$ in Fig. $2 \mathrm{~b}$ are s.d. of $k_{\text {obs }}$ values obtained from these two independent replicates.

For decapping assays measuring kinetics with $\mathrm{G}$ versus $\mathrm{C}$ at the first transcribed nucleotide described in Fig. 4 and those measuring $K_{\mathrm{M}}$ and $k_{\max }$ for Edcl activation (Supplementary Fig. 5, Supplementary Table 1), synthetic 5'-triphosphate 29-mer RNA oligonucleotides with either guanosine (G-RNA) or cytosine (C-RNA) as the $5^{\prime}$ nucleotide were obtained from Trilink BioTechnologies and enzymatically capped with ${ }^{32} \mathrm{P}$-radiolabeled GTP, as above. In the decapping reaction, $S p$ Dcp1-Dcp2(1-243) had a final concentration ranging from $2 \mu \mathrm{M}$ to $4 \mathrm{nM}$, the final concentration of $S p \operatorname{Edcl}(155-180)$ peptide was held constant at $50 \mu \mathrm{M}$, and capped RNA concentration was $<100 \mathrm{pM}$. For these experiments, glycerol was removed from the decapping buffer described above in order to obtain more easily saturable kinetics with the 29-mer RNA substrate. Decapping reactions were prepared, executed, and analyzed as described above. To obtain $k_{\max }$ and $K_{\mathrm{M}}, k_{\mathrm{obs}}$ was plotted versus protein concentration and fit to the model: $k_{\mathrm{obs}}=k_{\max }[\mathrm{E}] / K_{\mathrm{M}}+$ [E]. Errors on $k_{\text {obs }}$ values shown in Fig. 4 a are s.d. of two independent replicates. Errors on $k_{\max }$ values shown in Fig. $4 \mathrm{~b}$ are the standard error from fits to determine $k_{\max }$ and $K_{\mathrm{M}}$.

Data availability. Coordinates and structure factors were deposited in the Protein Data Bank with accession code PDB 6AM0. Other data are available from the corresponding author upon reasonable request.
Received: 25 August 2017 Accepted: 22 February 2018

Published online: 20 March 2018

\section{References}

1. Moore, M. J. From birth to death: the complex lives of eukaryotic mRNAs. Science 309, 1514-1518 (2005).

2. Parker, R. RNA degradation in Saccharomyces cerevisae. Genetics 191, 671-702 (2012)

3. Dunckley, T. \& Parker, R. The DCP2 protein is required for mRNA decapping in Saccharomyces cerevisiae and contains a functional MutT motif. EMBO J. 18, 5411-5422 (1999).

4. Arribas-Layton, M., Wu, D., Lykke-Andersen, J. \& Song, H. Structural and functional control of the eukaryotic mRNA decapping machinery. Biochim. Biophys. Acta 580-589, 2013 (1829).

5. Li, Y. \& Kiledjian, M. Regulation of mRNA decapping. Wiley Interdiscip. Rev. 1, 253-265 (2010)

6. Nagarajan, V. K., Jones, C. I., Newbury, S. F. \& Green, P. J. XRN 5' $\rightarrow 3^{\prime}$ exoribonucleases: structure, mechanisms and functions. Biochim. Biophys. Acta 1829, 590-603 (2013).

7. Kervestin, S. \& Jacobson, A. NMD: a multifaceted response to premature translational termination. Nat. Rev. Mol. Cell Biol. 13, 700-712 (2012).

8. Popp, M. W.-L. \& Maquat, L. E. Organizing principles of mammalian nonsense-mediated mRNA decay. Annu. Rev. Genet. 47, 139-165 (2013).

9. Cho, H., Kim, K. M. \& Kim, Y. K. Human proline-rich nuclear receptor coregulatory protein 2 mediates an interaction between mRNA surveillance machinery and decapping complex. Mol. Cell 33, 75-86 (2009).

10. Karousis, E. D., Nasif, S. \& Mühlemann, O. Nonsense-mediated mRNA decay: novel mechanistic insights and biological impact. Wiley Interdiscip. Rev. RNA 7, 661-682 (2016)

11. Guo, H., Ingolia, N. T., Weissman, J. S. \& Bartel, D. P. Mammalian microRNAs predominantly act to decrease target mRNA levels. Nature 466, 835-840 (2010).

12. Jonas, S. \& Izaurralde, E. Towards a molecular understanding of microRNAmediated gene silencing. Nat. Rev. Genet. 16, 421-433 (2015).

13. Geisler, S., Lojek, L., Khalil, A. M., Baker, K. E. \& Coller, J. Decapping of long non-coding RNAs regulates inducible genes. Mol. Cell 45, 279-291 (2012).

14. Presnyak, V. et al. Codon optimality is a major determinant of mRNA stability. Cell 160, 1111-1124 (2015).

15. Mauer, J. et al. Reversible methylation of m6Am in the $5^{\prime}$ cap controls mRNA stability. Nature 541, 371-375 (2017).

16. She, M. et al. Crystal structure and functional analysis of Dcp2p from Schizosaccharomyces pombe. Nat. Struct. Mol. Biol. 13, 63-70 (2006)

17. She, M. et al. Structural basis of Dcp2 recognition and activation by Dcp1. Mol. Cell 29, 337-349 (2008).

18. Floor, S. N., Borja, M. S. \& Gross, J. D. Interdomain dynamics and coactivation of the mRNA decapping enzyme Dcp2 are mediated by a gatekeeper tryptophan. Proc. Natl. Acad. Sci. USA 109, 2872-2877 (2012).

19. Wurm, J. P., Holdermann, I., Overbeck, J. H., Mayer, P. H. O. \& Sprangers, R Changes in conformational equilibria regulate the activity of the Dcp2 decapping enzyme. Proc. Natl. Acad. Sci. USA 114, 6034-6039 (2017).

20. Valkov, E. et al. Structure of the Dcp2-Dcp1 mRNA-decapping complex in the activated conformation. Nat. Struct. Mol. Biol. 23, 574-579 (2016)

21. Mugridge, J. S., Ziemniak, M., Jemielity, J. \& Gross, J. D. Structural basis of mRNA-cap recognition by Dcp1-Dcp2. Nat. Struct. Mol. Biol. 23, 987-994 (2016).

22. Charenton, C. et al. Structure of the active form of Dcp1-Dcp2 decapping enzyme bound to m7GDP and its Edc3 activator. Nat. Struct. Mol. Biol. 23 982-986 (2016)

23. Coller, J. mRNA decapping in 3D. Nat. Struct. Mol. Biol. 23, 954 (2016).

24. Valkov, E., Jonas, S. \& Weichenrieder, O. Mille viae in eukaryotic mRNA decapping. Curr. Opin. Struct. Biol. 47, 40-51 (2017).

25. Piccirillo, C., Khanna, R. \& Kiledjian, M. Functional characterization of the mammalian mRNA decapping enzyme hDcp2. RNA 9, 1138-1147 (2003).

26. Deshmukh, M. V. et al. mRNA decapping is promoted by an RNA-binding channel in Dcp2. Mol. Cell 29, 324-336 (2008).

27. Aglietti, R. A., Floor, S. N., McClendon, C. L., Jacobson, M. P. \& Gross, J. D. Active site conformational dynamics are coupled to catalysis in the mRNA decapping enzyme Dcp2. Structure 21, 1571-1580 (2013).

28. Mildvan, A. S. et al. Structures and mechanisms of Nudix hydrolases. Arch. Biochem. Biophys. 433, 129-143 (2005).

29. He, F. \& Jacobson, A. Control of mRNA decapping by positive and negative regulatory elements in the Dcp2 C-terminal domain. RNA 21, 1633-1647 (2015).

30. Fromm, S. A. et al. The structural basis of Edc3- and Scd6-mediated activation of the Dcp1:Dcp2 mRNA decapping complex. EMBO J. 31, 279-290 (2012). 
31. Floor, S. N., Jones, B. N., Hernandez, G. A. \& Gross, J. D. A split active site couples cap recognition by Dcp2 to activation. Nat. Struct. Mol. Biol. 17, 1096-1101 (2010).

32. Chang, C.-T., Bercovich, N., Loh, B., Jonas, S. \& Izaurralde, E. The activation of the decapping enzyme DCP2 by DCP1 occurs on the EDC4 scaffold and involves a conserved loop in DCP1. Nucleic Acids Res. 42, 5217-5233 (2014).

33. Jonas, S. \& Izaurralde, E. The role of disordered protein regions in the assembly of decapping complexes and RNP granules. Genes Dev. 27, 2628-2641 (2013).

34. Dunckley, T., Tucker, M. \& Parker, R. Two related proteins, Edclp and Edc2p, stimulate mRNA decapping in Saccharomyces cerevisiae. Genetics 157, 27-37 (2001).

35. Borja, M. S., Piotukh, K., Freund, C. \& Gross, J. D. Dep1 links coactivators of mRNA decapping to Dcp2 by proline recognition. RNA 17, 278-290 (2011).

36. Lai, T. et al. Structural basis of the PNRC2-mediated link between mRNA surveillance and decapping. Structure 20, 2025-2037 (2012).

37. Wurm, J. P., Overbeck, J. \& Sprangers, R. The S. pombe mRNA decapping complex recruits cofactors and an Edc1-like activator through a single dynamic surface. RNA 22, 1360-1372 (2016).

38. Kshirsagar, M. \& Parker, R. Identification of Edc3p as an enhancer of mRNA decapping in Saccharomyces cerevisiae. Genetics 166, 729-739 (2004).

39. Badis, G., Saveanu, C., Fromont-Racine, M. \& Jacquier, A. Targeted mRNA degradation by deadenylation-independent decapping. Mol. Cell 15, 5-15 (2004).

40. Dong, S. et al. YRA1 autoregulation requires nuclear export and cytoplasmic Edc3p-mediated degradation of its pre-mRNA. Mol. Cell 25, 559-573 (2007).

41. Harigaya, Y., Jones, B. N., Muhlrad, D., Gross, J. D. \& Parker, R. Identification and analysis of the interaction between Edc3 and Dcp2 in Saccharomyces cerevisiae. Mol. Cell. Biol. 30, 1446-1456 (2010).

42. Paquette, D. R., Tibble, R. W., Daifuku, T. S. \& Gross, J. D. Control of mRNA decapping by autoinhibition. Preprint at https://doi.org/10.1101/266718 (2018).

43. Fenger-Grøn, M., Fillman, C., Norrild, B. \& Lykke-Andersen, J. Multiple processing body factors and the ARE binding protein TTP activate mRNA decapping. Mol. Cell 20, 905-915 (2005).

44. Yu, J. H., Yang, W.-H., Gulick, T., Bloch, K. D. \& Bloch, D. B. Ge-1 is a central component of the mammalian cytoplasmic mRNA processing body. RNA 11, 1795-1802 (2005).

45. Eulalio, A. et al. Target-specific requirements for enhancers of decapping in miRNA-mediated gene silencing. Genes Dev. 21, 2558-2570 (2007).

46. Gavin, A.-C. et al. Functional organization of the yeast proteome by systematic analysis of protein complexes. Nature 415, 141-147 (2002)

47. Krogan, N. J. et al. Global landscape of protein complexes in the yeast Saccharomyces cerevisiae. Nature 440, 637-643 (2006).

48. Collins, S. R. et al. Functional dissection of protein complexes involved in yeast chromosome biology using a genetic interaction map. Nature 446, 806-810 (2007).

49. Decourty, L. et al. Linking functionally related genes by sensitive and quantitative characterization of genetic interaction profiles. Proc. Natl. Acad. Sci. USA 105, 5821-5826 (2008).

50. Ziemniak, M. et al. Two-headed tetraphosphate cap analogs are inhibitors of the Dcpl/2 RNA decapping complex. RNA 22, 518-529 (2016).

51. Lin, J., Abeygunawardana, C., Frick, D. N., Bessman, M. J. \& Mildvan, A. S. Solution structure of the quaternary MutT-M2+-AMPCPP $-\mathrm{M} 2+$ complex and mechanism of its pyrophosphohydrolase action. Biochemistry $\mathbf{3 6}$ 1199-1211 (1997).

52. Jones, B. N., Quang-Dang, D.-U., Oku, Y. \& Gross, J. D. in Methods in Enzymology (eds Maquat, L.E. and Kiledjian, M.) Vol. 448, 23-40 (Academic Press, Cambridge, Massachusetts, 2008).

53. Höfer, K. et al. Structure and function of the bacterial decapping enzyme NudC. Nat. Chem. Biol. 12, 730-734 (2016).

54. Li, H. et al. Genome-wide analysis of core promoter structures in Schizosaccharomyces pombe with DeepCAGE. RNA Biol. 12, 525-537 (2015).

55. Carninci, P. et al. Genome-wide analysis of mammalian promoter architecture and evolution. Nat. Genet. 38, 626-635 (2006).

56. Ziemniak, M. et al. Phosphate-modified analogues of $\mathrm{m}(7) \mathrm{GTP}$ and $\mathrm{m}(7)$ Gppppm(7)G-synthesis and biochemical properties. Bioorg. Med. Chem. 23, 5369-5381 (2015).

57. Kabsch, W. XDS. Acta Crystallogr. D Biol. Crystallogr. 66, 125-132 (2010).

58. McCoy, A. J. et al. Phaser crystallographic software. J. Appl. Crystallogr. 40, 658-674 (2007).
59. Adams, P. D. et al. PHENIX: a comprehensive Python-based system for macromolecular structure solution. Acta Crystallogr. D Biol. Crystallogr. 66, 213-221 (2010)

60. Emsley, P., Lohkamp, B., Scott, W. G. \& Cowtan, K. Features and development of Coot. Acta Crystallogr. D Biol. Crystallogr. 66, 486-501 (2010).

61. Chen, V. B. et al. MolProbity: all-atom structure validation for macromolecular crystallography. Acta Crystallogr. D Biol. Crystallogr. 66, 12-21 (2010).

62. Dolinsky, T. J. et al. PDB2PQR: expanding and upgrading automated preparation of biomolecular structures for molecular simulations. Nucleic Acids Res. 35, W522-W525 (2007).

63. Baker, N. A., Sept, D., Joseph, S., Holst, M. J. \& McCammon, J. A. Electrostatics of nanosystems: application to microtubules and the ribosome. Proc. Natl. Acad. Sci. USA 98, 10037-10041 (2001).

64. Krissinel, E. \& Henrick, K. Inference of macromolecular assemblies from crystalline state. J. Mol. Biol. 372, 774-797 (2007).

\section{Acknowledgements}

We thank J. Holton and G. Meigs at Lawrence Berkeley National Laboratory, Advanced Light Source beamline 8.3.1, and X. Liu for help with X-ray data collection and refinement. We also thank J. Kowalska for helpful discussions on the design and synthesis of the two-headed cap analog. This work was supported by the US National Institutes of Health (R01 GM078360 to J.D.G. and NRSA fellowship F32 GM105313 to J.S.M.), The Foundation for Polish Science (TEAM/2016-2/13 to J.J.), the National Science Centre, Poland (fellowship no. UMO-2014/12/T/NZ1/00528 to M.Z.), the Genentech Foundation Fellowship (to R.W.T), and the UCSF Discovery Fellowship (to R.W.T). The Advanced Light Source is supported by the US Department of Energy under contract no. DE-AC0205CH11231.

\section{Author contributions}

J.S.M. designed and purified all protein constructs, carried out crystallization experiments, collected and refined crystallographic data, wrote the manuscript, and prepared the figures. J.S.M. and R.W.T. carried out decapping kinetics experiments. M.Z. and J.J. designed and synthesized the two-headed cap analog. J.D.G. supervised the project and experimental design and guided manuscript preparation and editing. All authors read and commented on the manuscript.

\section{Additional information}

Supplementary Information accompanies this paper at https://doi.org/10.1038/s41467 018-03536-x.

Competing interests: The authors declare no competing interests.

Reprints and permission information is available online at http://npg.nature.com/ reprintsandpermissions/

Publisher's note: Springer Nature remains neutral with regard to jurisdictional claims in published maps and institutional affiliations.

Open Access This article is licensed under a Creative Commons Attribution 4.0 International License, which permits use, sharing, adaptation, distribution and reproduction in any medium or format, as long as you give appropriate credit to the original author(s) and the source, provide a link to the Creative Commons license, and indicate if changes were made. The images or other third party material in this article are included in the article's Creative Commons license, unless indicated otherwise in a credit line to the material. If material is not included in the article's Creative Commons license and your intended use is not permitted by statutory regulation or exceeds the permitted use, you will need to obtain permission directly from the copyright holder. To view a copy of this license, visit http://creativecommons.org/ licenses/by/4.0/.

(c) The Author(s) 2018 